\title{
Progress and challenges in improving surgical outcomes
}

\section{J. D. Birkmeyer}

Center for Healthcare Outcomes and Policy, University of Michigan, Building 520, Room 3145, 2800 Plymouth Road, Ann Arbor, Michigan 48109, USA (e-mail: jbirkmey@umich.edu)

Presented to the Annual Meeting of the European Surgical Association, Hamburg, Germany, May 2012

Published online in Wiley Online Library (www.bjs.co.uk). DOI: 10.1002/bjs.8933

Surgical morbidity and mortality are rightly considered public health concerns. It has been estimated that more than 200 hundred million major surgical procedures are performed annually worldwide ${ }^{1}$. Risks vary widely, related to the procedure involved, as well as patient and provider factors. Across these clinically diverse populations, at least a million patients die and an order of magnitude more experience serious complications after surgery every year.

Fortunately, recent evidence from the USA suggests trends toward improvement. Despite largely flat mortality rates for most high-risk cancer and cardiovascular procedures during the $1990 \mathrm{~s}^{2}$, risks associated with these procedures began to fall steadily after the turn of the millennium ${ }^{3}$. These trends cannot be explained by case mix. In fact, most reports indicate that patients undergoing major inpatient procedures have become older and, by most measures, less healthy over time. Technological innovation may help explain declining mortality for some procedures, such as endovascular repair of abdominal aortic aneurysms, but for many procedures, basic surgical techniques have changed little in the past two decades.

So why is surgery becoming safer? In the simplest terms, there are two basic mechanisms for improving patient outcomes: direct patients to hospitals and surgeons with the best results, and improve care everywhere. With regard to the former, the past decade has seen significant concentration of complex cancer procedures in many Westernized healthcare systems. In the USA hundreds of low-volume hospitals stopped performing procedures such as pancreatectomy and oesophagectomy, and median hospital volumes rose sharply ${ }^{3}$. Redistribution of surgical patients to higher-volume, lowermortality hospitals was a significant factor underlying declining mortality for many cancer operations. It, nevertheless, explained less than half of the overall effect. Concentration of patients and expertise played no role in safer cardiovascular surgery. Mortality after cardiac and peripheral vascular procedures declined just as much as that after cancer surgery, despite trends toward fewer overall procedures dispersed across an increasing number of hospitals in the USA.

Such evidence indicates that surgical mortality is falling at hospitals across the entire performance spectrum. Their respective contributions remain speculative, but several factors may be responsible.

\section{Heightened awareness}

It is perhaps no coincidence that the seminal report from the Institute of Medicine, To Err is Human: Building a Safer Healthcare System, was issued in 1999, around the same time as surgical mortality rates began to fall ${ }^{4}$. Highlighting 44000-98000 deaths each year from medical errors prompted unprecedented scrutiny of hospital safety, with possible 'trickle down' effects on a safety culture, levels of staffing and other aspects of surgical care.

\section{Outcomes measurement}

Although initiated at various times, clinical registries, institutional and national audits, providing regular performance feedback to hospitals and surgeons, have been launched by several specialty societies, local and regional health agencies and national health ministries. A growing literature supports the idea of a 'surgical Hawthorne effect', whereby the act of performance measurement and feedback leads to improved outcomes in advance of specific, measurable changes in practice. In northern New England, for example, the mortality rate after coronary artery bypass grafting fell by more than 25 percent within 6 months after feedback of mortality data to hospitals and surgeons ${ }^{5}$. After implementation of the National Surgical Quality Improvement Program, surgical morbidity rates in Department of Veterans Affairs hospitals fell by over 40 per cent in 2 years ${ }^{6}$.

\section{Performance-related payment}

Beginning in the early 2000s, many payers began providing financial rewards (payment by results) to providers for compliance with evidencebased practices associated with reduced complications, including surgical-site infection and venous thromboembolism. Current research in both surgery and general medical 
practice suggests that such programmes are often successful in increasing compliance with targeted practices, but do little to improve outcomes $^{7}$. These data indicate the complexity of high-quality surgical care and suggest that focusing on a shortlist of measurable processes of care is insufficient.

\section{Checklists}

Following successful checklist interventions to reduce catheter-related bloodstream infections ${ }^{8}$, two large studies demonstrated significant reductions in surgical morbidity and mortality after implementation of comprehensive checklists during and/or after surgery ${ }^{9,10}$. In one of the studies, outcomes improvement was largely unrelated to how compliant hospitals were with the specific components of the checklists ${ }^{9}$, implying that checklists may exert their salutary effects by inspiring teamwork, communication and a culture of safety, rather than through the direct effect of the specific processes of care they target.

\section{Getting to the next level of surgical safety}

In some respects, many of the above improvement strategies target 'lowhanging fruit'. Checklists help surgeons to avoid making simple mistakes, such as surgery at the wrong site. Payment for performance leads to more consistent perioperative care of proven benefit. Further improvements will require a much deeper understanding of mechanisms underlying variation in provider performance and levers for improving not only perioperative care but also what the surgeon does in the operating room.

Rather than 'one-size-fits-all' guidelines for perioperative care, these should, in future, be tailored to the specific clinical context and sometimes to the individual patient. Prophylaxis against surgical complications should, for example, be datadriven. The Michigan Bariatric Surgical Collaborative used data from a large statewide outcomes registry to develop a regression-based prediction model for identifying patients at low, medium, and high risk of venous thromboembolism, the leading cause of death after bariatric surgery. Optimal strategies for medical prophylaxis were identified for patients in each risk stratum and then implemented across all 32 hospitals in Michigan. Within a year, rates of venous thromboembolism and mortality had fallen by over half, to levels substantially below national benchmarks ${ }^{11}$.

Although avoiding complications is crucial, timeliness of interventions and the proficiency with which patients are managed after adverse events occur should not be overlooked. In one national study from the USA, hospitals with low and high mortality rates had virtually indistinguishable rates of postoperative complications $^{12}$. The hospital groups differed primarily in their 'failure to rescue' rates, that is how often patients died once a serious complication occurred. Further research will need to elucidate the types of resources and aspects of safety culture and practice necessary for minimizing failure to rescue.

Getting to the next level of surgical safety also requires improving what happens in the operating room, not just afterwards. Studies of human factors have highlighted how distractions and problems with teamwork and communication among operating room team members lead to errors ${ }^{13}$. Improving surgical outcomes may also mean paying greater attention to the proficiency of the operating surgeon. A large body of literature linking volume and training to outcomes provides at least indirect evidence of variation in surgeon skill. Unpublished research from the author's centre indicates that surgeons' technical skill, as assessed by blinded peer ratings, varies widely and correlates strongly with complication rates in bariatric surgery. These data suggest that optimizing surgical outcomes after some procedures may require better tools for measuring and improving the performance of surgeons themselves, not just the systems in which they operate.

\section{Disclosure}

The author declares no conflict of interest.

\section{References}

1 Weiser TG, Regenbogen SE, Thompson KD, Haynes AB, Lipsitz SR, Berry WR et al. An estimation of the global volume of surgery: a modeling strategy based on available data. Lancet 2008; 372 : 139-144.

2 Goodney P, Siewers A, Stukel TA, Birkmeyer JD. Is cancer surgery getting safer? National trends in operative mortality. 7 Am Coll Surg 2002; 195: 219-227.

3 Finks JF, Osborne NH, Birkmeyer JD. Trends in hospital volume and operative mortality for high-risk surgery. N Engl 7 Med 2011; 364: 2128-2137.

4 Kohn LT, Corrigan JM, Donaldson MS (eds). To Err is Human: Building a Safer Healthcare System. Institute of Medicine, National Academies Press: Washington, 2000.

5 O'Connor GT, Plume SK, Olmstead EM, Morton JR, Maloney CT, Nugent WC et al. A regional intervention to improve the hospitalmortality associated with coronary artery bypass graft surgery. The Northern New England 
Cardiovascular Disease Study Group. 7AMA 1996; 275: 841-846.

6 Khuri SF, Daley J, Henderson WG. The comparative assessment and improvement of quality of surgical care in the Department of Veterans Affairs. Arch Surg 2002; 137: 20-27.

7 Nicholas LH, Osborne NH, Birkmeyer JD, Dimick JB. Hospital process compliance and surgical outcomes in medicare beneficiaries. Arch Surg 2010; 145: 999-1004.

8 Pronovost PJ, Needham D, Berenholtz S, Sinopoli D, Chu H, Cosgrove $\mathrm{S}$ et al. An intervention to decrease catheter-related bloodstream infections in the ICU. N Engl 7 Med 2006; 355: 2725-2732.

9 Haynes AB, Weiser TG, Berry WR, Lipsitz SR, Breizat AH, Dellinger EP et al.; Safe Surgery Saves Lives Study Group. A surgical safety checklist to reduce morbidity and mortality in a global population. N Engl F Med 2009; 360: 491-499.

10 de Vries EN, Prins HA, Crolla R, den Outer AJ, van Andel G, van Helden SH et al.; SURPASS Collaborative Group. Effect of a comprehensive surgical safety system on patient outcomes. $N$ Engl 7 Med 2010; 363: 1928-1937.
11 Share DA, Campbell DA, Birkmeyer N, Prager RL, Gurm HS, Moscucci $M$ et al. How a regional collaborative of hospitals and physicians in Michigan cut costs and improved the quality of care. Health Aff (Millwood) 2011; 30: 636-645.

12 Ghaferi AA, Birkmeyer JD, Dimick JB. Variation in hospital mortality with inpatient surgery. $N$ Engl 7 Med 2009; 361: 1368-1375.

13 Vincent C, Moorthy K, Sarker SK, Chang A, Darzi AW. Systems approaches to surgical quality and safety: from concept to measurement. Ann Surg 2004; 239: 475-482. 